\title{
NEVANLINNA-TYPE CHARACTERIZATIONS FOR THE BLOCH SPACE AND RELATED SPACES
}

\author{
by KAREL STROETHOFF \\ (Received 16th August 1988)
}

\begin{abstract}
We give a characterisation of the Bloch space in terms of an area version of the Nevanlinna characteristic, analogous to Baernstein's description of the space $B M O A$ in terms of the usual Nevanlinna characteristic. We prove analogous results for the little Bloch space and the space $V M O A$, and give value distribution characterizations for all these spaces. Finally we give valence conditions on a Bloch or little Bloch function for containment in $B M O A$ or $V M O A$.
\end{abstract}

1980 Mathematics subject classification (1985 Revision): 30H05, 30D35, 30 C99.

\section{Introduction}

Our starting point is Baernstein's [3] result that an analytic function on the unit disk belongs to the space of analytic functions of bounded mean oscillation, $B M O A$, if and only if its Möbius-transforms form a bounded family in the Nevanlinna class. In this paper we give similar characterizations for related spaces of analytic functions such as $V M O A$, the Bloch space and the little Bloch space; these spaces are defined in Section 1. In Section 2 we give a description of $V M O A$ in terms of the Nevanlinna characteristic. A description of VMOA cannot be obtained by simply replacing Baernstein's boundedness condition by the corresponding vanishing condition (as is usually the case). In Section 3 we describe the Bloch space and little Bloch space in terms of the pseudohyperbolic metric on the unit disk. We then formulate and prove characterizations for these spaces in terms of an area version of the Nevanlinna characteristic in Section 4. In Sections 5 and 6 we give value distribution characterizations for $V M O A$ and the Bloch spaces. In the final section, Section 7 , we prove necessary conditions for a function in the Bloch space or the little Bloch space to belong to $B M O A$ or $V M O A$. The results in this paper give further support to the idea that the Bloch space and the little Bloch space can be considered as area versions of the spaces $B M O A$ and $V M O A$, respectively.

\section{Definitions and notation}

Let $\mathbb{D}=\{z \in \mathbb{C}:|z|<1\}$ denote the open unit disk in the complex plane. Let $\partial \mathbb{D}=$ $\{z \in \mathbb{C}:|z|=1\}$ denote the unit circle, and let $m$ denote the normalized Lebesgue measure on $\partial \mathbb{D}$. Recall the definition of the Hardy spaces: for an analytic function $f$ on $\mathbb{D}$ and $0<p<\infty$, define $\|f\|_{H^{p}}=\left(\sup _{0<r<1} \int_{\partial D}|f(r \zeta)|^{p} d m(\zeta)\right)^{1 / p}$. The Hardy space $H^{p}$ is 
defined to be the set of all analytic functions $f$ on the disk $\mathbb{D}$ for which $\|f\|_{H^{D}}<\infty$. If $f \in H^{p}$, for $0<p<\infty$, then the radial limit $f^{*}(\zeta)=\lim _{r \rightarrow 1}-f(r \zeta)$ exists for [m]-a.e. $\zeta \in \partial \mathbb{D}$, and the function $f^{*}$ belongs to $L^{p}(\partial \mathbb{D}, m)([4$, Theorem 2.2]).

Suppose $g \in L^{1}(\partial \mathbb{D}, m)$. For an arc $I$ in $\partial \mathbb{D}$ let $g_{I}$ denote the average of $g$ over $I$ : $g_{I}=m(I)^{-1} \int_{I} g d m$; put $\|g\|_{B M O}=\sup \left\{m(I)^{-1} \int_{I}\left|g-g_{I}\right| d m: I\right.$ an arc in $\left.\partial \mathbb{D}\right\}$. A function $g$ in $L^{1}(\partial \mathbb{D}, m)$ for which $\|g\|_{B M O}<\infty$ is said to be of bounded mean oscillation. The set of all functions in $L^{1}(\partial \mathbb{D}, m)$ that are of bounded mean oscillation is denoted by $B M O$. The class $B M O$ was first introduced by John and Nirenberg in [7] (in the context of functions defined on cubes in $\left.\mathbb{R}^{n}\right)$. Define $B M O A=\left\{f \in H^{1}: f^{*} \in B M O\right\}$, and for $f \in B M O A$ set $\|f\|_{B M O A}=\left\|f^{*}\right\|_{B M O}$. Equipped with the norm $\|f\|_{B M O A}+|f(0)|, B M O A$ is a Banach space.

For $\lambda \in \mathbb{D}$ let the Möbius function $\varphi_{\lambda}: \mathbb{D} \rightarrow \mathbb{D}$ be defined by

$$
\varphi_{\lambda}(z)=\frac{\lambda-z}{1-\bar{\lambda} z}, \quad z \in \mathbb{D} .
$$

For an analytic function $f$ on $\mathbb{D}$ and a point $\lambda \in \mathbb{D}$, we will call the function $f \circ \varphi_{\lambda}-f(\lambda)$ a Möbius transform of function $f$.

Two quantities $A_{f}$ and $B_{f}$, both depending on an analytic function $f$ on $\mathbb{D}$, are said to be equivalent, written as $A_{f} \approx B_{f}$, if there exists a finite positive constant $C$ not depending on $f$ such that for every analytic function $f$ on $\mathbb{D}$ we have: $C^{-1} B_{f} \leqq A_{f} \leqq$ $C B_{f}$. If the quantities $A_{f}$ and $B_{f}$ are equivalent, then in particular $A_{f}<\infty$ if and only if $B_{f}<\infty$.

The space $B M O A$ is invariant under Möbius transforms, i.e., if $f \in B M O A$ and $\lambda \in \mathbb{D}$, then $f \circ \varphi_{\lambda}-f(\lambda) \in B M O A$. In fact, for $0<p<\infty$ it can be shown (see, for example, [13]) that for every analytic function $f$ on $\mathbb{D}$ :

$$
\|f\|_{B M O A} \approx \sup _{\lambda \in \mathbb{D}}\left\|f \circ \varphi_{\lambda}-f(\lambda)\right\|_{H^{p}}
$$

In [12] Sarason introduced the space $V M O$ of functions of vanishing mean oscillation defined by $V M O=\left\{g \in L^{1}(\partial \mathbb{D}, m): m(I)^{-1} \int_{I}\left|g-g_{I}\right| d m \rightarrow 0\right.$ as $\left.m(I) \rightarrow 0\right\}$. Define $V M O A=$ $\left\{f \in H^{1}: f^{*} \in V M O\right\}$. Since clearly $V M O$ is contained in $B M O$, we have that $V M O A$ is contained in $B M O A$. It can be shown (see, for example, [13]) that analogous to equivalence (1.2), if $0<p<\infty$, then for every analytic function $f$ on $\mathbb{D}$ :

$$
f \in V M O A \Leftrightarrow\left[\left\|f \circ \varphi_{\lambda}-f(\lambda)\right\|_{H^{p} \rightarrow 0} \text { as }|\lambda| \rightarrow 1^{-}\right] .
$$

Another Möbius-invariant Banach space is the Bloch space. For an analytic function $f$, on $\mathbb{D}$ we set $\|f\|_{\mathscr{Z}}=\sup \left\{\left(1-|z|^{2}\right)\left|f^{\prime}(z)\right|: z \in \mathbb{D}\right\}$. The Bloch space $\mathscr{B}$ is the set of all analytic functions $f$ on $\mathbb{D}$ for which $\|f\|_{\mathscr{T}}<\infty$. Even though $\|\cdot\|_{\boxplus}$ is not a norm, we will refer to $\|f\|_{\circledast \rightarrow}$ as the Bloch norm of function $f$. The quantity $|f(0)|+\|f\|_{\infty}$ defines a norm on the linear space $\mathscr{B}$ which, equipped with this norm, is a Banach space (see, for example, [1]). That the Bloch space is invariant under Möbius transforms follows 
immediately from the definition of the Bloch norm: for an analytic function $f$ on $\mathbb{D}$ and $\lambda \in \mathbb{D}$ it is easy to verify that $\|f\|_{\mathscr{B}}=\left\|f \circ \varphi_{\lambda}\right\|_{\mathscr{B}}$. In [11] Rubel and Timoney showed that the Bloch space $\mathscr{B}$ is in some sense maximal among all Möbius-invariant Banach spaces of analytic functions on $\mathbb{D}$.

Contained in the Bloch space is the little Bloch space $\mathscr{B}_{0}$, which is by definition the set of all analytic functions $f$ on $\mathbb{D}$ for which $\left(1-|z|^{2}\right) f^{\prime}(z) \rightarrow 0$ as $|z| \rightarrow 1^{-}$.

Using Taylor series it is easy to see that $\left|f^{\prime}(0)\right| \leqq\|f\|_{H^{2}}$ for every analytic function $f$ on $\mathbb{D}$. It follows that for an analytic function $f$ on $\mathbb{D}$ and a point $\lambda \in \mathbb{D}$ :

$$
\left(1-|\lambda|^{2}\right)\left|f^{\prime}(\lambda)\right| \leqq\left\|f \circ \varphi_{i}-f(\lambda)\right\|_{H^{2}}
$$

Thus we have the inclusions $B M O A \subset \mathscr{B}$ and $V M O A \subset \mathscr{B}_{0}$.

Let $A$ denote the usual Lebesgue area measure on the complex plane $\mathbb{C}$. For an analytic function $f$ on $\mathbb{D}$ and $0<p<\infty$ we define $\|f\|_{L_{\alpha}^{p}}=\left(\int_{\mathbb{D}}|f|^{p} d A / \pi\right)^{1 / p}$. The Bergman space $L_{a}^{p}$ is defined to be the set of all analytic functions $f$ on $\mathbb{D}$ for which $\|f\|_{L_{a}^{p}}<\infty$. The subscript $a$ stands for "analytic". In analogy to (1.2), for $0<p<\infty$ it can be shown (see [2]) that for every analytic function $f$ on $\mathbb{D}$ :

$$
\|f\|_{\mathscr{S A}} \approx \sup _{\lambda \in \mathbb{D}}\left\|f \circ \varphi_{\lambda}-f(\lambda)\right\|_{L_{a}}
$$

The analogue of (1.3) is that for $0<p<\infty$ for every analytic function $f$ on $\mathbb{D}$ :

$$
f \in \mathscr{B}_{0} \Leftrightarrow\left\|f \circ \varphi_{i}-f(\lambda)\right\|_{L_{a}^{p}} \rightarrow 0 \quad \text { as } \quad|\lambda| \rightarrow 1^{-}
$$

For $f$ on analytic $\mathbb{D}$ the Nevanlinna characteristic $T(f)$ is defined by

$$
T(f)=\sup _{0 \leqq r<1} \frac{1}{2 \pi} \int_{0}^{2 \pi} \log { }^{+}\left|f\left(r e^{i \theta}\right)\right| d \theta .
$$

\section{The space $V M O A$ in terms of the Nevanlinna characteristic}

In this section we describe the space $V M O A$ in terms of the Nevanlinna characteristic. Our starting point in Baernstein's characterization for the space $B M O A$; he proved that an analytic function on the unit disk belongs to the space $B M O A$ if and only if the Möbius transforms of the function form a bounded family in the Nevanlinna class. We give a similar description of the space VMOA. This description cannot be obtained by simply replacing Baernstein's boundedness condition by the corresponding vanishing condition (as is usually the case).

Let $0<p<\infty$, then it follows from the inequality $p \log ^{+} x \leqq x^{p}$ that

$$
p \frac{1}{2 \pi} \int_{0}^{2 \pi} \log { }^{+}\left|f\left(r e^{i \theta}\right)\right| d \theta \leqq \frac{1}{2 \pi} \int_{0}^{2 \pi}\left|f\left(r e^{i \theta}\right)\right|^{p} d \theta
$$


hence

$$
T(f) \leqq \frac{1}{p}\|f\|_{H^{p}}^{p}, \quad \text { for } \quad 0<p<\infty
$$

Using equivalence (1.2) it follows from (2.2) that for $f \in B M O A$, $\sup _{\lambda \in \mathbb{D}} T\left(f \circ \varphi_{\lambda}-f(\lambda)\right)<\infty$, i.e., the family $\left\{f \circ \varphi_{\lambda}-f(\lambda): \lambda \in \mathbb{D}\right\}$ is bounded in the Nevanlinna class $N=\{f: f$ analytic on $\mathbb{D}$ and $T(f)<\infty\}$. In [3] Baernstein proved that the converse is also true. Before stating his result we need to introduce more notation. Fix $0<\alpha<\pi / 2$. For $e^{i \theta} \in \partial \mathbb{D D}$ let $\Gamma_{\alpha}\left(e^{i \theta}\right)$ denote the Stolz region based at $e^{i \theta}$, i.e., $\Gamma_{\alpha}\left(e^{i \theta}\right)$ is the interior of the convex hull of the circle $|z|=\sin \alpha$ and the point $e^{i \theta}$. The non-tangential maximal function $\mathcal{N}_{\alpha}(f)$ of a complex function $f$ defined on $\mathbb{D}$ is defined by

$$
\left(\mathcal{N}_{\alpha}(f)\right)\left(e^{i \theta}\right)=\sup \left\{|f(z)|: z \in \Gamma_{\alpha}\left(e^{i \theta}\right)\right\} .
$$

Note that $\left(\mathscr{N}_{\alpha}(f)\right)\left(e^{i \theta}\right) \geqq\left|f^{*}\left(e^{i \theta}\right)\right|$ if $f$ has a non-tangential limit $f^{*}\left(e^{i \theta}\right)$ at $e^{i \theta}$. In [3] Baernstein proved the following "John-Nirenberg type" of theorem:

Theorem 2.1 (Baernstein [3]). There exists an absolute constant $K$ such that for each $0<\alpha<\pi / 2$ and $f$ analytic on $\mathbb{D}$ the following statements are equivalent:

(A) $\left\{f \circ \varphi_{\lambda}-f(\lambda): \lambda \in \mathbb{D}\right\}$ is bounded in the Nevanlinna class $N$;

(B) There exists a constant $\beta=\beta(\alpha, f)$ for which

$$
m\left(\left\{e^{i \theta}: \mathcal{N}_{\alpha}\left(f \circ \varphi_{\lambda}-f(\lambda)\right)\left(e^{i \theta}\right)>t\right\}\right)<K e^{-\beta t},
$$

for all $\lambda \in \mathbb{D}$, and for all $0<t<\infty$.

As Baernstein indicated ([3, Corollary 5.2]), Theorem 2.1 has as an immediate consequence:

Theorem 2.2 (Baernstein [3]). For an analytic function $f$ on $\mathbb{D}$ the following statements are equivalent:

(A) $f \in B M O A$;

(B) $\sup _{\lambda \in \mathbb{D}} T\left(f \circ \varphi_{\lambda}-f(\lambda)\right)<\infty$.

What about the space $V M O A$ ? One may be tempted to replace the above big-O condition (B) in Theorem 2.2 by the corresponding little-o condition, and ask whether

$$
f \in V M O A \Leftrightarrow T\left(f \circ \varphi_{\lambda}-f(\lambda)\right) \rightarrow 0 \quad \text { as } \quad|\lambda| \rightarrow 1^{-} ?
$$


The answer is negative: the condition at the right of (2.4) is certainly necessary for $f$ to be in VMOA (this follows from (1.3) and (2.2)), but not sufficient. That the condition is not sufficient follows from the observation that it is trivially satisfied when $\|f\|_{\infty} \leqq 1 / 2$ (because this implies that $T\left(f \circ \varphi_{\lambda}-f(\lambda)\right)=0$ for all $\left.\lambda \in \mathbb{D}\right)$, but not every analytic function $f$ on $\mathbb{D}$ for which $\|f\|_{\infty} \leqq 1 / 2$ is contained in $V M O A$.

Let us return to $B M O A$ and rewrite the condition in Theorem 2.2. If $\rho>0$ and $f \in B M O A$, then also $f / \rho \in B M O A$, so that $\sup _{\lambda \in \mathbb{D}} T\left(\left(f \circ \varphi_{\lambda}-f(\lambda)\right) / \rho\right)<\infty$. It follows that for $f$ analytic on $\mathbb{D}$ :

$$
f \in B M O A \Leftrightarrow\left[\forall \rho>0: \sup _{\lambda \in \mathbb{D}} T\left(\left(f \circ \varphi_{\lambda}-f(\lambda)\right) / \rho\right)<\infty\right] .
$$

Having replaced the big-O condition in Theorem 2.2 by a collection of big-O conditions in (2.5), going to the corresponding little-o conditions yields the following:

Theorem 2.3. For an analytic function $f$ on $\mathbb{D}$ the following statements are equivalent:

(a) $f \in V M O A$;

(b) for every $\rho>0$ we have that $T\left(\left(f \circ \varphi_{\lambda}-f(\lambda)\right) / \rho\right) \rightarrow 0$ as $|\lambda| \rightarrow 1^{-}$.

Before the proof we need to relate the Nevanlinna characteristic and the $H^{2}$-norm of an analytic function. We'll do this not just for the $H^{2}$-norm, but for any $H^{p}$-norm:

Lemma 2.4. Let $0<p<\infty$. For an analytic function $f$ on $\mathbb{D}$ :

$$
\|f\|_{H^{p}}=p^{2} \int_{0}^{\infty} \rho^{p-1} T(f / \rho) d \rho .
$$

Proof. Let $0<p<\infty$. For $0 \leqq x<\infty$ we have: $\int_{0}^{\infty} \rho^{p-1} \log ^{+}(x / \rho) d \rho=x^{p} / p^{2}$. Thus, for an analytic function $f$ on $\mathbb{D}$ and $0<r<1$ an application of Fubini's theorem gives:

$$
\int_{0}^{\infty} \rho^{p-1}\left(\frac{1}{2 \pi} \int_{0}^{2 \pi} \log { }^{+}\left|f\left(r e^{i \theta}\right) / \rho\right| d \theta\right) d \rho=\frac{1}{p^{2}} \frac{1}{2 \pi} \int_{0}^{2 \pi}\left|f\left(r e^{i \theta}\right)\right|^{p} d \theta .
$$

Taking the limit as $r \rightarrow 1^{-}$, and using the Monotone Convergence Theorem we get (2.6).

Now we are ready for the proof of Theorem 2.3 .

Proof of Theorem 2.3. Let $f$ be an analytic function on $\mathbb{D}$. We have already seen that condition (b) in Theorem 2.3 is necessary.

To prove the sufficiency, suppose that $f$ satisfies condition (b). Our first step is in showing that $f \in B M O A$. Choose an $r \in(0,1)$ such that $T\left(f \circ \varphi_{\lambda}-f(\lambda)\right)<1$ whenever $r<|\lambda|<1$. Note that $g \in N \Leftrightarrow g \circ \varphi_{\lambda} \in N$ (this follows easily from the fact that each 
function in the Nevanlinna class $N$ is the quotient of two $H^{\infty}$-functions). Pick $w$ such that $r<|w|<1$. Then $T\left(f \circ \varphi_{w}-f(w)\right)<1$, so that $f \circ \varphi_{w} \in N$, and therefore $f \in N$. Thus $\log ^{+}|f|$ has a harmonic majorant, call it $h$. Then for $\lambda \in \mathbb{D}, h \circ \varphi_{\lambda}$ is a harmonic majorant of $\log ^{+}\left|f \circ \varphi_{\lambda}\right|$, whence $T\left(f \circ \varphi_{\lambda}\right) \leqq\left(h \circ \varphi_{\lambda}\right)(0)=h(\lambda)$. Using the inequality $\log ^{+}(x+y) \leqq \log ^{+} x+\log ^{+} y+\log 2$, it follows that for $|\lambda| \leqq r: T\left(f \circ \varphi_{\lambda}-f(\lambda)\right) \leqq h(\lambda)+$ $\log ^{+}|f(\lambda)|+\log 2$. Hence the family $\left\{f \circ \varphi_{\lambda}-f(\lambda): \lambda \in \mathbb{D}\right\}$ is bounded in $N$, and by Theorem 2.2 we have $f \in B M O A$.

Since $f \in B M O A$ we can apply Theorem 2.1. Let $\beta$ be such that (2.3) holds. Then for $\lambda \in \mathbb{D}$ and $t>0: m\left(\left\{e^{i \theta}:\left|f^{*}\left(\varphi_{\lambda}\left(e^{i \theta}\right)\right)-f(\lambda)\right|>t\right\}\right)<K e^{-\beta t}$. Using this inequality as well as the distribution function for the $\log ^{+}$, it follows that for every $\rho>0$ :

$$
\begin{aligned}
T\left(\left(f \circ \varphi_{\lambda}-f(\lambda)\right) / \rho\right) & \leqq \frac{1}{2 \pi} \int_{0}^{2 \pi} \log ^{+}\left(\left|f^{*}\left(\varphi_{\lambda}\left(e^{i \theta}\right)\right)-f(\lambda)\right| / \rho\right) d \theta \\
& =\int_{\rho}^{\infty} \frac{1}{t} m\left(\left\{e^{i \theta}:\left|f^{*}\left(\varphi_{\lambda}\left(e^{i \theta}\right)\right)-f(\lambda)\right|>t\right\}\right) d t \\
& \leqq \int_{\rho}^{\infty} \frac{1}{t} K e^{-\beta t} d t \leqq \frac{K}{\rho \beta} e^{-\beta \rho}
\end{aligned}
$$

Now let $\varepsilon>0$ be given. Choose $R>0$ such that $K e^{-\beta R}<\left(\varepsilon^{2} \beta^{2}\right) / 8$. Then integrating the above inequality we get

$$
\int_{R}^{\infty} \rho T\left(\left(f \circ \varphi_{\lambda}-f(\lambda)\right) / \rho\right) d \rho<\varepsilon^{2} / 8
$$

By the Lebesgue Dominated Convergence Theorem we can choose $\delta \in(0,1)$ such that

$$
\int_{0}^{R} \rho T\left(\left(f \circ \varphi_{\lambda}-f(\lambda)\right) / \rho\right) d \rho<\varepsilon^{2} / 8
$$

whenever $1-\delta<|\lambda|<1$. Using the formula of Lemma 2.4, it follows from (2.8) and (2.9) that

$$
\left\|f \circ \varphi_{\lambda}-f(\lambda)\right\|_{H^{2}}^{2}=4 \int_{0}^{\infty} \rho T\left(\left(f \circ \varphi_{\lambda}-f(\lambda)\right) / \rho\right) d \rho<\varepsilon^{2}
$$

hence $\left.\| f \circ \varphi_{\lambda}-f(\lambda)\right) \|_{H^{2}}<\varepsilon$, whenever $1-\delta<|\lambda|<1$. Therefore $f \in V M O A$, and the theorem is proved.

The classical Nevanlinna characteristic $T$ is defined in terms of $\log ^{+}$, which only 
measures the values of the function that are of modulus bigger than 1 . Instead we could define

$$
T^{\prime}(f)=\sup _{0 \leqq r<1} \frac{1}{2 \pi} \int_{0}^{2 \pi} \log \left(1+\left|f\left(r e^{i \theta}\right)\right|\right) d \theta
$$

for an analytic function $f$ on $\mathbb{D}$, and we obtain a characteristic equivalent to $T$. In fact, it is easy to show that for every analytic function $f$ on $\mathbb{D}, T(f) \leqq T^{\prime}(f) \leqq T(f)+\log 2$. The characteristic $T^{\prime}$ also measures values of the function that are of modulus less than 1. The following theorem is an easy consequence of Theorem 2.3 . We omit the proof.

Theorem 2.5. For an analytic function $f$ on $\mathbb{D}$ the following statements are equivalent:

(a) $f \in V M O A$;

(b) $T^{\prime}\left(f \circ \varphi_{\lambda}-f(\lambda)\right) \rightarrow 0$ as $|\lambda| \rightarrow 1^{-}$.

\section{The Bloch spaces and the pseudo-hyperbolic metric}

In this section we will give characterizations of the Bloch space and the little Bloch space in terms of the pseudo-hyberbolic metric on the unit disk. In the next section these results will be used to obtain analogues of the characterizations of $B M O A$ and $V M O A$ discussed in the previous section.

For points $\lambda, z$ in the disk $\mathbb{D}$ the pseudo-hyperbolic distance $d(\lambda, z)$ between $\lambda$ and $z$ is defined by $d(\lambda, z)=\left|\varphi_{\lambda}(z)\right|$. It it can be shown that $d$ is a metric on $\mathbb{D}$ (see, for example, $[5$, p. 4]). In this section we see that the Bloch space consists of those analytic functions on the disk that are uniformly continuous with respect to the pseudo-hyperbolic metric. We will give a similar description for the little Bloch space.

For each point $\lambda \in \mathbb{D}$ and $0<r<1$, the pseudo-hyperbolic disk $D(\lambda, r)$ with pseudohyperbolic centre $\lambda$ and pseudo-hyperbolic radius $r$ is defined by $D(\lambda, r)=\{z \in \mathbb{D}: d(\lambda, z)<$ $r\}=\left\{z \in \mathbb{D}:\left|\varphi_{\lambda}(z)\right|<r\right\}$. Note that $D(0, r)$ is equal to the euclidean disk $\{z \in \mathbb{D}:|z|<r\}$. Now, if $w=\varphi_{\lambda}(z)$, then, as is easily checked, $z=\varphi_{\lambda}(w)$ and so $D(\lambda, r)=\left\{\varphi_{\lambda}(w):|w|<r\right\}=$ $\varphi_{\lambda}(D(0, r))$. Consequently, the pseudo-hyperbolic disk $D(\lambda, r)$ is also a euclidean disk: it can be shown that its euclidean centre and euclidean radius are $\left(1-r^{2}\right) \lambda /\left(1-r^{2}|\lambda|^{2}\right)$ and $\left(1-|\lambda|^{2}\right) r /\left(1-r^{2}|\lambda|^{2}\right)$, respectively (see [5]).

First we will show that for an analytic function on the disk the Bloch norm and the supremum of the oscillations of the function over pseudo-hyperbolic disks of a fixed radius are equivalent quantities.

Theorem 3.1. Let $0<r<1$. For $f$ analytic on $\mathbb{D}$ the following quantities are equivalent:

(A) $\|f\|_{s ;}$

(B) $\sup _{\lambda \in \mathbf{D}} \sup _{z \in D(\lambda, r)}|f(z)-f(\lambda)|$. 
Proof. Fix $0<r<1$, and let $f$ be analytic on $\mathbb{D}$. It follows from the identity

$$
f^{\prime}(0)=\frac{2}{r^{4}} \int_{D(0, r)} \bar{z} f(z) d A(z) / \pi
$$

that

$$
\left|f^{\prime}(0)\right| \leqq \frac{2}{r} \sup _{z \in D(0, r)}|f(z)|
$$

Replacing $f$ by $f \circ \varphi_{\lambda}-f(\lambda)$, we get the inequality

$$
\left(1-|\lambda|^{2}\right)\left|f^{\prime}(\lambda)\right| \leqq \frac{2}{r} \sup _{z \in D(\lambda, r)}|f(z)-f(\lambda)|
$$

and it follows that

$$
\|f\|_{\mathscr{B}} \leqq \frac{2}{r} \sup _{\lambda \in \mathbb{D}} \sup _{z \in D(\lambda, r)}|f(z)-f(\lambda)|
$$

On the other hand for $|w|<r$ we have

$$
|f(w)-f(0)| \leqq \int_{0}^{1} \frac{|w|}{1-t^{2}|w|^{2}} d t \cdot\|f\|_{\mathscr{B}} \leqq \frac{1}{2} \log \left(\frac{1+r}{1-r}\right)\|f\|_{\mathscr{D}} .
$$

Replacing $f$ by $f \circ \varphi_{\lambda}-f(\lambda)$ yields

$$
\left|f\left(\varphi_{\lambda}(w)\right)-f(\lambda)\right| \leqq \frac{1}{2} \log \left(\frac{1+r}{1-r}\right)\|f\|_{\mathscr{A}}
$$

whenever $|w|<r$. Hence

$$
\sup _{\lambda \in \mathbb{D}} \sup _{z \in D(\lambda, r)}|f(z)-f(\lambda)| \leqq \frac{1}{2} \log \left(\frac{1+r}{1-r}\right)\|f\|_{\mathscr{B}}
$$

and the theorem is proved.

Remark. As a corollary of the proof of the above theorem we see that the Bloch space consists of those analytic functions on the unit disk that are uniformly continuous with respect to the pseudo-hyperbolic metric.

As usual, the equivalences of the previous theorem carry over to the little Bloch space. This is expressed in the following theorem. 
Theorem 3.2. Let $0<r<1$. For an analytic function $f$ on $\mathbb{D}$ the following statements are equivalent:

(a) $f \in \mathscr{B}_{0}$;

(b) $\sup _{z \in D(\lambda, r)}|f(z)-f(\lambda)| \rightarrow 0$ as $|\lambda| \rightarrow 1^{-}$.

Proof. That (b) implies (a) follows immediately from (3.1).

For the converse, suppose that $f \in \mathscr{B}_{0}$. For $t \in(0,1)$ the dilate $f_{t}$ is defined by $f_{t}(z)=f(t z)$ for $z \in \mathbb{D}$. By (3.2) we have for $t \in(0,1)$ and $\lambda \in \mathbb{D}$

$$
\sup _{z \in D(\lambda, r)}\left|f(z)-f_{t}(z)-\left(f(\lambda)-f_{t}(\lambda)\right)\right| \leqq \frac{1}{2} \log \left(\frac{1+r}{1-r}\right)\left\|f-f_{t}\right\|_{\mathscr{B}}
$$

Using the triangle inequality it follows that for $t \in(0,1)$ and $\lambda \in \mathbb{D}$

$$
\sup _{z \in D(\lambda, r)}|f(z)-f(\lambda)| \leqq \frac{1}{2} \log \left(\frac{1+r}{1-r}\right)\left\|f-f_{t}\right\|_{\mathscr{B}}+\sup _{z \in D(\lambda, r)}\left|f_{t}(z)-f_{f}(\lambda)\right| .
$$

For $t \in(0,1)$ fixed, the dilate $f_{t}$ is analytic in a neighbourhood of the disk, so that clearly $\sup \left\{\left|f_{t}(z)-f_{t}(\lambda)\right|: z \in D(\lambda, r)\right\} \rightarrow 0$ as $|\lambda| \rightarrow 1^{-}$. Since $f \in \mathscr{B}_{0}$, we have $\left\|f-f_{t}\right\|_{\mathscr{B}} \rightarrow 0$ as $t \rightarrow 1^{-}$ (see [1, Theorem 2.1]). Hence (b) follows easily from (3.3).

\section{Nevanlinna-type characterizations for the Bloch spaces}

In this section we formulate and prove characterizations for the Bloch space and the little Bloch space in terms of an area version of the Nevanlinna characteristic.

For $f$ analytic on $\mathbb{D}$ the area version of the Nevanlinna characteristic, $T_{a}(f)$, is defined by

$$
T_{a}(f)=\int_{0} \log ^{+}|f| d A / \pi
$$

The area-Nevanlinna class is the set $N_{a}=\left\{f \in H(\mathbb{D}): T_{a}(f)<\infty\right\}$. Let $0<p<\infty$. Integrating both sides of inequality (2.1) gives, in analogy to (2.2):

$$
T_{a}(f) \leqq \frac{1}{p}\|f\|_{L_{a}^{p}}^{p}, \quad \text { for } \quad 0<p<\infty
$$

So the area-Nevanlinna class contains all Bergman spaces. Analogous to Baernstein's 
characterization for the space $B M O A$ given in Theorem 2.2 we have the following result for the Bloch space:

Theorem 4.1. For an analytic function $f$ on $\mathbb{D}$ the following statements are equivalent:

(A) $f \in \mathscr{B}$;

(B) $\sup _{\lambda \in \mathbb{D}} T_{a}\left(f \circ \varphi_{\lambda}-f(\lambda)\right)<\infty$.

Proof. That (A) implies (B) follows from (4.1) and (1.5).

For the converse, let $f$ be an analytic function on $\mathbb{D}$ and suppose that (B) holds. Fix $0<r<1$, and let $z, \lambda \in \mathbb{D}$ with $d(z, \lambda)<r$. Put $u=\varphi_{\lambda}(z)$, then $|u|<r$ and $z=\varphi_{\lambda}(u)$. Using that the function $\log ^{+}\left|f \circ \varphi_{\lambda}-f(\lambda)\right|$ is subharmonic on $\mathbb{D}$ we have

$$
\begin{aligned}
\log ^{+}|f(z)-f(\lambda)| & =\log ^{+}\left|\left(f \circ \varphi_{\lambda}\right)(u)-f(\lambda)\right| \\
& \leqq \frac{1}{(1-r)^{2}} \int_{|w-u|<1-r} \log ^{+}\left|\left(f \circ \varphi_{\lambda}\right)(w)-f(\lambda)\right| d A(w) / \pi \\
& \leqq \frac{1}{(1-r)^{2}} T_{a}\left(f \circ \varphi_{\lambda}-f(\lambda)\right) \leqq \frac{M}{(1-r)^{2}}
\end{aligned}
$$

where $M$ is the quantity in (B). Since $x \leqq \exp \left(\log ^{+} x\right)$ for $x \geqq 0$, we have $|f(z)-f(\lambda)| \leqq$ $\exp \left(M /(1-r)^{2}\right)$, and it follows from Theorem 3.1 that $f \in \mathscr{B}$, as was to be shown.

A description of the little Bloch space in terms of the area-Nevanlinna characteristic is contained in the following theorem which is analogous to the description of the space $V M O A$ given in Theorem 2.3 .

Theorem 4.2. For an analytic function $f$ on $\mathbb{D}$ the following statements are equivalent:

(a) $f \in \mathscr{B}_{0}$;

(b) For every $\rho>0$ we have that $T_{a}\left(\left(f \circ \varphi_{\lambda}-f(\lambda)\right) / \rho\right) \rightarrow 0$ as $|\lambda| \rightarrow 1^{-}$.

Proof. That (b) is implied by (a) follows easily from (4.1) and (1.6).

For the converse, suppose that $f$ is an analytic function on $\mathbb{D}$ for which (b) holds. Fix $0<r<1$. Let $z, \lambda \in \mathbb{D}$ such that $d(z, \lambda)<r$. Then, as in the proof of Theorem 4.1:

$$
|f(z)-f(\lambda)| / \rho \leqq \exp \left\{(1-r)^{-2} T_{a}\left(\left(f \circ \varphi_{\lambda}-f(\lambda)\right) / \rho\right)\right\}
$$

Given $\varepsilon>0$, choose $0<\rho<\varepsilon / 2$. Since (b) holds we can choose a $\delta \in(0,1)$ for which $T_{a}\left(\left(f \circ \varphi_{\lambda}-f(\lambda)\right) / \rho\right)<(1-r)^{2} \log 2$ whenever $0<1-|\lambda|<\delta$. Combining this with (4.2) we 
see that for $0<1-|\lambda|<\delta, \quad|f(z)-f(\lambda)| \leqq 2 \rho<\varepsilon$. Thus $\sup \{\mid f(z|-f(\lambda)-f(z)|$ : $z \in D(\lambda, r)\} \rightarrow 0$ as $|\lambda| \rightarrow 1^{-}$, so that by Theorem $3.4, f \in \mathscr{B}_{0}$, and we are done.

\section{A value distribution characterization for the space $V M O A$}

In this section we will give a different proof of Baernstein's value distribution characterization for $B M O A([3$, Theorem 3$])$ and then formulate and prove the corresponding description for the space VMOA.

The Green's function for the unit disk $\mathbb{D}$ is given by $G(z, \lambda)=\log \left(1 /\left|\varphi_{\lambda}(z)\right|\right)$, for $z, \lambda \in \mathbb{D}$. For a nonconstant analytic function $f$ on $\mathbb{D}$ let $\left\{z_{n}(f)\right\}$ denote the zeros of $f$ in $\mathbb{D}$, listed in increasing moduli and repeated according to multiplicities. Following Baernstein we define $N(w, \lambda, f)$, the "counting function for value $w$ started at $\lambda$ ", by

$$
N(w, \lambda, f)=\sum_{n} G\left(z_{n}(f-w), \lambda\right)
$$

Note that $G(z, 0)=\log (1 /|z|)$, so that

$$
N(w, 0, f)=\sum_{n} \log \frac{1}{\left|z_{n}(f-w)\right|},
$$

the usual counting function. It is clear from the definition of the counting function that

$$
\begin{gathered}
N(w, \lambda, f)=\infty \quad \text { if } f(\lambda)=w \\
N(w, \lambda, f)=0 \quad \text { if } f \text { omits the value } w .
\end{gathered}
$$

The following properties of the counting function, which are easily verified, are useful: For $w \in \mathbb{C}, \alpha \in \mathbb{C} \backslash\{0\}, \lambda \in \mathbb{D}$ and $f$ analytic on $\mathbb{D}$ we have:

$$
\begin{gathered}
N(w, \lambda, f)=N(w+\alpha, \lambda, f+\alpha) \\
N(w, \lambda, f)=N(\alpha w, \lambda, \alpha f) \\
N(w, \lambda, f)=N\left(0,0, f \circ \varphi_{\lambda}-w\right) .
\end{gathered}
$$

The following theorem is due to Baernstein ([3, Theorem 3]). We will give a simpler proof of his theorem.

Theorem 5.1 (Baernstein [3]). For a nonconstant analytic function $f$ on $\mathbb{D}$ the following statements are equivalent:

(A) $f \in B M O A$;

(B) $\sup \{N(w, \lambda, f): w \in \mathbb{C}, \lambda \in \mathbb{D}$ and $|f(\lambda)-w| \geqq 1\}<\infty$. 
Just as in Baernstein's proof we will need to relate the Nevanlinna characteristic of an analytic function with its counting function. This is done in the following classical result.

Cartan's Formula. For a nonconstant analytic function $f$ on $\mathbb{D}$ :

$$
T(f)=\frac{1}{2 \pi} \int_{0}^{2 \pi} N\left(e^{i \theta}, 0, f\right) d \theta+\log ^{+}|f(0)| .
$$

A proof of Cartan's Formula can be found in [6, pp. 214-215], for the case that $f$ is analytic on a neighbourhood of $\mathbb{D}$. The general case follows easily by looking at the dilates $f_{t}$ of $f$. Using the Monotone Convergence Theorem we see that $T\left(f_{t}\right)$ increases to $T(f)$ and for each $\theta$ in $(0,2 \pi)$ we have that $N\left(e^{i \theta}, 0, f_{t}\right)$ increases to $N\left(e^{i \theta}, 0, f\right)$ as we take the limit $t \rightarrow 1^{-}$. For these dilates $f_{t}$ we know that (5.3) holds, so that another application of the Monotone Convergence Theorem gives that (5.3) holds for $f$.

Proof of Theorem 5.1. Let $f$ be a nonconstant analytic function on $\mathbb{D}$. By Jensen's Formula we have:

$$
\frac{1}{2 \pi} \int_{0}^{2 \pi} \log \left|f\left(r e^{i \theta}\right)\right| d \theta=\sum_{n:\left|z_{n}(f)\right|<r} \log \frac{r}{\left|z_{n}(f)\right|}+\log |f(0)| .
$$

Thus

$$
\frac{1}{2 \pi} \int_{0}^{2 \pi} \log { }^{+}\left|f\left(r e^{i \theta}\right)\right| d \theta \geqq \sum_{n:\left|z_{n}(f)\right|<r} \log \frac{r}{\left|z_{n}(f)\right|}+\log |f(0)|,
$$

which after taking the limit $r \rightarrow 1^{-}$, gives us the inequality

$$
T(f) \geqq N(0,0, f)+\log |f(0)| \text {. }
$$

Replacing $f$ by $f \circ \varphi_{\lambda}-w$, and making use of (5.2c) the above inequality yields $N(w, \lambda, f) \leqq T\left(f \circ \varphi_{\lambda}-w\right)-\log |f(\lambda)-w|$. Using the inequality $\log ^{+}(x+y) \leqq \log ^{+} x+$ $\log ^{+} y+\log 2$, we get $N(w, \lambda, f) \leqq T\left(f \circ \varphi_{\lambda}-f(\lambda)\right)+\log ^{+}|f(\lambda)-w|-\log |f(\lambda)-w|+\log 2$. So if $|f(\lambda)-w| \geqq 1$, then we have $N(w, \lambda, f) \leqq T\left(f \circ \varphi_{\lambda}-f(\lambda)\right)+\log 2$. This inequality and Theorem 2.2 show that (A) implies (B).

To prove the converse suppose that $M=\sup \{N(w, \lambda, f): w \in \mathbb{C}, \lambda \in \mathbb{D}$ and $|f(\lambda)-w| \geqq 1\}<\infty$. By Cartan's Formula

$$
T\left(f \circ \varphi_{\lambda}-f(\lambda)\right)=\frac{1}{2 \pi} \int_{0}^{2 \pi} N\left(e^{i \theta}, 0, f \circ \varphi_{\lambda}-f(\lambda)\right) d \theta .
$$

Now, using (5.2a) and (5.2c), for every $0 \leqq \theta \leqq 2 \pi$ we have $N\left(e^{i \theta}, 0, f \circ \varphi_{\lambda}-f(\lambda)\right)=$ $N\left(e^{i \theta}+f(\lambda), \lambda, f\right) \leqq M$, so it follows that $T\left(f \circ \varphi_{\lambda}-f(\lambda)\right) \leqq M$, for all $\lambda \in \mathbb{D}$, and hence, by Theorem 2.2, $f \in B M O A$. 
Before going to $V M O A$ let us rewrite the condition in Theorem 5.1 for inclusion in $B M O A$. Suppose that $f \in B M O A$, and let $\delta>0$. Since $f / \delta \in B M O A$, it satisfies condition (B) of Theorem 5.1. By $(5.2 \mathrm{~b}), N(w, \lambda, f)=N(w / \delta, \lambda, f / \delta)$. Therefore we must have that for an analytic function $f$ on $\mathbb{D}$ :

$$
f \in B M O A \Leftrightarrow[\forall \delta>0: \sup \{N(w, \lambda, f): w \in \mathbb{C}, \lambda \in \mathbb{D} \text { and }|f(\lambda)-w| \geqq \delta\}<\infty] \text {. }
$$

We will show that the little-o condition corresponding to the big-O condition in (5.5) will give a necessary and sufficient condition for inclusion in the space $V M O A$. This will be made precise in Theorem 5.3.

In the proof of Theorem 5.3 we will need to relate the counting function $N$ of an analytic function to the $H^{2}$-norm of the function. As is shown in the following lemma, this can be done not just for the $H^{2}$-norm but for any $H^{p}$-norm of an analytic function.

Lemma 5.2. Let $0<p<\infty$. For an analytic function $f$ on $\mathbb{D}$ with $f(0)=0$ :

$$
\|f\|_{H^{p}}^{p}=\frac{p^{2}}{2 \pi} \int_{C}|w|^{p-2} N(w, 0, f) d A(w)
$$

Proof. Fix $0<p<\infty$, and let $f$ be an analytic function on $\mathbb{D}$ with $f(0)=0$. By Cartan's Formula and (5.2b), for every $\rho>0$ :

$$
T(f / \rho)=\frac{1}{2 \pi} \int_{0}^{2 \pi} N\left(e^{i \theta}, 0, f / \rho\right) d \theta=\frac{1}{2 \pi} \int_{0}^{2 \pi} N\left(\rho e^{i \theta}, 0, f\right) d \theta .
$$

Multiply by $\rho^{p-1}$ and integrate with respect to $\rho$ over the interval $(0, \infty)$. By formula (2.6) of Lemma 2.4 we get

$$
\begin{aligned}
\|f\|_{H^{p}}^{p} & =p^{2} \int_{0}^{\infty} \rho^{p-1}\left(\frac{1}{2 \pi} \int_{0}^{2 \pi} N\left(\rho e^{i \theta}, 0, f\right) d \theta\right) d \rho \\
& =\frac{p^{2}}{2 \pi} \int_{\mathrm{C}}|w|^{p-2} N(w, 0, f) d A(w),
\end{aligned}
$$

and the lemma is proved.

Theorem 5.3. For a nonconstant analytic function $f$ on $\mathbb{D}$ the following statements are equivalent:

(a) $f \in V M O A$;

(b) for every $\delta>0$ we have: 
$\sup \{N(w, \lambda, f): w \in \mathbb{C}$ and $|f(\lambda)-w| \geqq \delta\} \rightarrow 0$ as $|\lambda| \rightarrow 1^{-}$.

Proof. Let $f$ be a nonconstant analytic function on $\mathbb{D}$. Let $\delta>0$. As in the proof of Theorem 5.1, using Cartan's Formula and the equations (5.2) we see

$$
\begin{aligned}
T\left(\left(f \circ \varphi_{\lambda}-f(\lambda)\right) / \delta\right) & =\frac{1}{2 \pi} \int_{0}^{2 \pi} N\left(\delta e^{i \theta}+f(\lambda), \lambda, f\right) d \theta \\
& \leqq \sup \{N(w, \lambda, f): w \in \mathbb{C} \text { and }|f(\lambda)-w| \geqq \delta\},
\end{aligned}
$$

so that, by Theorem 2.3, (b) implies (a).

To prove the other implication we make use of Lemma 5.2. In this lemma take $p=2$, and replace $f$ by $f \circ \varphi_{\lambda}-f(\lambda)$, we get the formula

$$
\left\|f \circ \varphi_{\lambda}-f(\lambda)\right\|_{H^{2}}^{2}=\frac{2}{\pi} \int_{C} N(w+f(\lambda), \lambda, f) d A(w) .
$$

We will also need Lehto's Theorem [8], which states that for a function $g$, analytic on a neighbourhood of $\mathbb{D}$, the function $w \mapsto N(w, 0, g)$ is subharmonic on $\mathbb{C} \backslash\{g(0)\}$. Let $g$ be an analytic function on $\mathbb{D}$ for which $g(0)=0$. Let $0<r<1$. Applying Lehto's theorem to the dilate $g_{r}$ of $g$ we get that for $\delta>0$ and for $|u| \geqq \delta$

$$
N\left(u, 0, g_{r}\right) \leqq \frac{1}{\pi \delta^{2}} \int_{|u-v|<\delta} N\left(v, 0, g_{r}\right) d A(v)
$$

Taking the limit where $r \rightarrow 1^{-}$, we get

$$
N(u, 0, g) \leqq \frac{1}{\pi \delta^{2}} \int_{|u-v|<\delta} N(v, 0, g) d A(v) .
$$

Apply the inequality to $g=f \circ \varphi_{\lambda}-f(\lambda)$. Using equations (5.2) we get

$$
N(u+f(\lambda), \lambda, f) \leqq \frac{1}{\pi \delta^{2}} \int_{|u-v|<\delta} N(v+f(\lambda), \lambda, f) d A(v)
$$

Replacing $u+f(\lambda)$ by $w$ yields the formula

$$
N(w, \lambda, f) \leqq \frac{1}{\pi \delta^{2}} \int_{|w-z|<\delta} N(z, \lambda, f) d A(z) .
$$

Combining (5.6) and (5.8) gives us that $\sup \{N(w, \lambda, f): w \in \mathbb{C}$ and $|f(\lambda)-w| \geqq \delta\} \leqq\left(1 / 2 \delta^{2}\right)$ $\left\|f \circ \varphi_{\lambda}-f(\lambda)\right\|_{H^{2}}^{2}$, from which it follows that (a) implies (b). 


\section{Value distribution characterizations for the Bloch and little Bloch space}

Now we will turn to the Bloch space and the little Bloch space. Defining an area version of the counting function used in the value distribution characterizations for $B M O A$ and $V M O A$, we obtain analogous results for the Bloch space and the little Bloch space.

Define an area version $N_{a}$ of the counting function $N$ as follows: given an analytic function $f$ on $\mathbb{D}$ we first define $N_{a}(0,0, f)$ by

$$
N_{a}(0,0, f)=\int_{0}^{1} 2 r N\left(0,0, f_{r}\right) d r
$$

and, mimicking (5.2c), for $w \in \mathbb{C}$ and $\lambda \in \mathbb{D}$ define $N_{a}(w, \lambda, f)$ by

$$
N_{a}(w, \lambda, f)=N_{a}\left(0,0, f \circ \varphi_{\lambda}-w\right)
$$

Observe that $N_{a}(w, \lambda, f)=0$ if $f$ omits the value $w$, but that (5.1a) is not necessarily true for counting function $N_{a}$. It follows immediately from the definition that properties (5.2) do hold for counting function $N_{a}$ : for $w \in \mathbb{C}, \alpha \in \mathbb{C} \backslash\{0\}, \lambda \in \mathbb{D}$ and $f$ analytic on $\mathbb{D}$ we have:

$$
\begin{gathered}
N_{a}(w, \lambda, f)=N_{a}(w+\alpha, \lambda, f+\alpha) \\
N_{a}(w, \lambda, f)=N_{a}(\alpha w, \lambda, \alpha f) \\
N_{a}(w, \lambda, f)=N_{a}\left(0,0, f \circ \varphi_{\lambda}-w\right) .
\end{gathered}
$$

Analogous to Baernstein's value distribution characterization for $B M O A$ (Theorem 5.1) we have the following result for the Bloch space.

Theorem 6.1. For a nonconstant analytic function $f$ on $\mathbb{D}$ the following statements are equivalent:

(A) $f \in \mathscr{B}$;

(B) $\sup \left\{N_{a}(w, \lambda, f): w \in \mathbb{C}, \lambda \in \mathbb{D}\right.$ and $\left.|f(\lambda)-w| \geqq 1\right\}<\infty$.

Proof. Let $f$ be a nonconstant analytic function on $\mathbb{D}$, and let $0<r<1$. By inequality (5.4) we have: $N\left(0,0, f_{r}\right) \leqq T\left(f_{r}\right)-\log |f(0)|$. Multiply this inequality by $2 r$ and integrate with respect to $r$ over the interval $(0,1)$ to get:

$$
N_{a}(0,0, f) \leqq T_{a}(f)-\log |f(0)| .
$$

Just as in the proof of Theorem 5.1 it follows that if $|f(\lambda)-w| \geqq 1$, then $N_{a}(w, \lambda, f) \leqq$ $T_{a}\left(f \circ \varphi_{\lambda}-f(\lambda)\right)+\log 2$. Theorem 4.1 and this inequality show that $(\mathrm{A})$ implies (B). 
Note that integrating Cartan's Formula gives us the formula

$$
T_{a}(f)=\frac{1}{2 \pi} \int_{0}^{2 \pi} N_{a}\left(e^{i \theta}, 0, f\right) d \theta+\log ^{+}|f(0)| .
$$

To prove the converse we use this formula and proceed as in the proof of Theorem 5.1.

The value distribution characterization for the Bloch space carries over to the little Bloch space in the same way as going from $B M O A$ to $V M O A$.

Theorem 6.2. For a nonconstant analytic function $f$ on $\mathbb{D}$ the following statements are equivalent:

(a) $f \in \mathscr{B}_{0}$;

(b) for every $\delta>0$ we have:

$$
\sup \left\{N_{a}(w, \lambda, f): w \in \mathbb{C} \text { and }|f(\lambda)-w| \geqq \delta\right\} \rightarrow 0 \text { as }|\lambda| \rightarrow 1^{-} \text {. }
$$

Proof. Let $f$ be a nonconstant analytic function on $\mathbb{D}$. Let $\delta>0$. Making use of (6.3) and the equations (6.1), as in the proof of Theorem 5.3, we have for every $\delta>0$ $T_{a}\left(\left(f \circ \varphi_{\lambda}-f(\lambda)\right) / \delta\right) \leqq \sup \left\{N_{a}(w, \lambda, f): w \in \mathbb{C}\right.$ and $\left.|f(\lambda)-w| \geqq \delta\right\}$, so that, by Theorem 4.2, (b) implies (a).

To prove the other implication we need an area-version of Lemma 5.2. If $0<p<\infty$, the function $f$ is analytic on $\mathbb{D}$, and $f(0)=0$, then applying Lemma 5.2 to the dilates $f_{r}$ of $f$ and subsequently integrating with respect to $r$ over the interval $(0,1)$ yields the formula

$$
\|f\|_{L_{a}^{p}}=\frac{p^{2}}{2 \pi} \int_{C}|w|^{p-2} N_{a}(w, 0, f) d A(w)
$$

In the above formula take $p=2$ and for $\lambda \in \mathbb{D}$ replace $f$ by $f \circ \varphi_{\lambda}-f(\lambda)$; analogous to (5.6) we get:

$$
\left\|f \circ \varphi_{\lambda}-f(\lambda)\right\|_{L_{a}^{2}}^{2}=\frac{2}{\pi} \int_{\mathrm{c}} N_{a}(w+f(\lambda), \lambda, f) d A(w)
$$

Integrating (5.7) with respect to $r$ over the interval $(0,1)$ gives that for an analytic function $g$ on $\mathbb{D}$ for which $g(0)=0$ and for $|u| \geqq \delta>0$ we have

$$
N_{a}(u, 0, g) \leqq \frac{1}{\pi \delta^{2}} \int_{|u-v|<\delta} N_{a}(v, 0, g) d A(v)
$$


As in the proof of Theorem 5.3 it follows that whenever $|f(\lambda)-w| \geqq \delta$ we must have

$$
N_{a}(w, \lambda, f) \leqq \frac{1}{\pi \delta^{2}} \int_{|w-z|<\delta} N_{a}(z, \lambda, f) d A(z)
$$

Combining (6.4) and (6.5) we get $\sup \left\{N_{a}(w, \lambda, f): w \in \mathbb{C}\right.$ and $\left.|f(\lambda)-w| \geqq \delta\right\} \leqq$ $\left(1 / 2 \delta^{2}\right)\left\|f \circ \varphi_{\lambda}-f(\lambda)\right\|_{L_{a}^{2}}^{2}$, from which it follows that (a) implies (b).

\section{Valence conditions for containment in $B M O A$ or $V M O A$}

For a nonconstant analytic function $f$ on $\mathbb{D}$ and $0<r<1$ let $n(f, r)$ denote the number of zeros of $f$ in the disk $D(0, r)$, counted according to multiplicities. Then $n(f)=\lim _{r \rightarrow 1}-n(f, r)$ denotes the number of times (counting multiplicities) that $f$ assumes the value 0. In [9] Pommerenke showed that a Bloch function $f$ which satisfies the valence condition

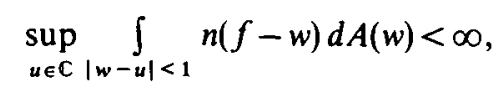

must belong to $B M O A$. If $f$ is univalent (or finitely-valent), then it is trivial that the above condition is satisfied, thus univalent (or finitely-valent) Bloch functions belong to $B M O A$. The following theorem gives a necessary and sufficient condition on a Bloch function for inclusion in the space $B M O A$.

Theorem 7.1. For a function $f \in \mathscr{B}$ the following statements are equivalent:

(A) $f \in B M O A$;

(B) $\sup \left\{\int_{0}^{1} \operatorname{tn}\left(f \circ \varphi_{\lambda}-w, t\right) d t: \lambda \in \mathbb{D}, w \in \mathbb{C}\right.$ and $\left.|f(\lambda)-w| \geqq 1\right\}<\infty$.

Remark. Note that $n\left(f \circ \varphi_{\lambda}-w, t\right)$ is the number of zeros of $f-w$ in the pseudohyperbolic disk $D(\lambda, t)$, counted according to multiplicities. Thus the above condition (B) is trivially satisfied if $f$ is univalent (or finitely-valent).

Proof. It is elementary to show that for an analytic function $F$ on $\mathbb{D}$ for which $F(0) \neq 0$

$$
N\left(0,0, F_{r}\right)=\int_{0}^{r} \frac{n(F, t)}{t} d t
$$

Thus it follows that

$$
N_{a}(0,0, F)=\int_{0}^{1}\left(2 r \int_{0}^{r} \frac{n(F, t)}{t} d t\right) d r=\int_{0}^{1}\left(\frac{1}{t}-t\right) n(F, t) d t
$$


Hence

$$
N_{a}(0,0, F)+\int_{0}^{1} \operatorname{tn}(F, t) d t=N(0,0, F)
$$

Take $\lambda \in \mathbb{D}$ and $w \in \mathbb{C}$, such that $|f(\lambda)-w| \geqq 1$. The analytic function $F=f \circ \varphi_{\lambda}-w$ satisfies $F(0) \neq 0$, so that the above formula yields

$$
N_{a}(w, \lambda, f)+\int_{0}^{1} \operatorname{tn}\left(f \circ \varphi_{\lambda}-w, t\right) d t=N(w, \lambda, f)
$$

which (in view of Theorems 5.1 and 6.1) immediately gives the result.

It is not immediately clear that condition (7.1) implies statement (B) in Theorem 7.1. It is however not difficult to see that (7.2), (5.8) and (6.4) imply that the supremum in statement (B) of Theorem 7.1 is less than or equal $(1 / 2) \sup _{\lambda \in \mathrm{D}}\left\|f \circ \varphi_{\lambda}-f(\lambda)\right\|_{L_{a}^{2}}^{2}+$ $(1 / \pi) \sup _{u \in C} \int_{|w-u|<1} n(f-w) d A(w)$, so that Pommerenke's result follows from Theorem 7.1 .

Using Theorems 5.2 and 6.2 we see from (7.2):

Theorem 7.2. For a function $f \in \mathscr{B}_{0}$ the following statements are equivalent:

(a) $f \in V M O A$;

(b) for every $\delta>0$ :

$$
\sup \left\{\int_{0}^{1} \operatorname{tn}\left(f \circ \varphi_{\lambda}-w, t\right) d t: \lambda \in \mathbb{D}, w \in \mathbb{C} \text { and }|f(\lambda)-w| \geqq \delta\right\} \rightarrow 0 \text { as }|\lambda| \rightarrow 1^{-} \text {. }
$$

Acknowledgement. This paper constitutes part of the author's doctoral dissertation written at Michigan State University under direction of Sheldon Axler. I would like to thank him for his guidance and encouragement.

\section{REFERENCES}

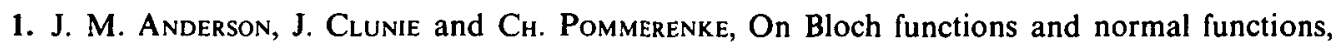
J. Reine Angew. Math. 270 (1974).

2. S. AxLER, The Bergman space, the Bloch space, and commutators of multiplication operators, Duke Math. J. 53 (1986), 315-332.

3. A. Baernstein, Analytic functions of bounded mean oscillation, in Aspects of Contemporary Complex Analysis (Eds. D. A. Brannan and J. G. Clunie, Academic Press, London, 1980), pp. 3-36.

4. P. L. Duren, Theory of $H^{p}$ Spaces (Academic Press, New York, 1970). 
5. J. B. Garnett, Bounded Analytic Functions (Academic Press, New York, 1981).

6. E. Hitle, Analytic Function Theory, Vol. II (Ginn and Company, New York, 1962).

7. F. John and L. Nirenberg, On functions of bounded mean oscillation, Comm. Pure Appl. Math. 14 (1961), 415-426.

8. O. Lehto, A majorant principle in the theory of functions, Math. Scand. 1 (1953), 1-17.

9. $\mathrm{C}_{\mathrm{H}}$. Pommerenke, Schlichte Funktionen und analytische Funktionen von beschränkter mittlerer Oszillation, Comment. Math. Helv. 52 (1977), 591-602.

10. Сh. Pommerenke, On univalent functions, Bloch functions and VMOA, Math. Ann. 236 (1978), 199-208.

11. L. A. Rubel and R. M. Timoney, An extremal property of the Bloch space, Proc. Amer. Math. Soc. 75 (1979), 45-49.

12. D. Sarason, Functions of vanishing mean oscillation, Trans. Amer. Math. Soc. 207 (1975), 391-405.

13. D. Sarason, Function Theory on the Unit Circle (Lecture notes of Virginia Polytechnic Institute and State University, Blacksburg, Virginia, 1978).

Department of Mathematical Sciences

UNIVERSITY OF MONTANA

Missoula, Montana 59812, USA 